NOTE

\title{
Use of multiple layers of Gafchromic film to increase sensitivity
}

\author{
Tsang Cheung ${ }^{1}$, Martin J Butson ${ }^{1,2,3}$ and Peter K N Yu ${ }^{1}$ \\ ${ }^{1}$ Department of Physics and Materials Science, City University of Hong Kong, Kowloon Tong, \\ Hong Kong, People's Republic of China \\ 2 Illawarra Cancer Care Centre, Department of Medical Physics, Crown St, Wollongong 2500, \\ NSW, Australia \\ E-mail: mbutson@usa.net
}

Received 6 February 2001, in final form 26 July 2001

Published 20 September 2001

Online at stacks.iop.org/PMB/46/N235

\begin{abstract}
This note investigates the increase of the sensitivity of radiochromic film by layering the film together. Investigations with up to five layers of radiochromic film placed together as a single dosimeter have allowed accurate two-dimensional dosimetry at low radiotherapy doses. The sensitivity of the response of the layered film increases with the number of layers, with the fivelayer dosimeter increasing the change in optical density by $4.3 \pm 0.2$ times that for a single layer film at $670 \mathrm{~nm}$ readout wavelength. Measurements in the penumbral region of a $6 \mathrm{MV}$ x-ray beam show that the spatial resolution is slightly reduced with the layered film. The distance across the region from 20 to $80 \%$ of the maximum dose at the beam edge (20/80\% penumbra) measured at $1.5 \mathrm{~cm}$ depth was measured as $3.1 \pm 0.3$ and $3.5 \pm 0.3 \mathrm{~mm}$ for one and five layers, respectively. Thus no major reduction in spatial resolution was found by layering the film to five layers but the sensitivity was increased by $430 \%$ at $670 \mathrm{~nm}$ readout wavelength.
\end{abstract}

\section{Introduction}

Gafchromic MD-55-2 film, due to its relatively low energy dependence compared to radiographic film, has become a significant dosimetry tool in high-energy radiotherapy (Klassen et al 1997, Niroomand-Rad et al 1998, McLaughlin et al 1991). One of its shortcomings for use in both in-phantom and in vivo dosimetry, however, has been its low sensitivity, normally requiring large doses (5 Gy or more) to produce acceptable results. There seem to be some discrepancies in the actual absorption spectral sensitivity of the MD-55-2 film reported within the literature. McLaughlin et al (1996) show the spectral response for the MD-55-2 film from 400 to $750 \mathrm{~nm}$ with doses to the film of 0, 10 and $60 \mathrm{~Gy}$. At the (approx $676 \mathrm{~nm}$ ) peak the change in absorbance is approximately 0.06 OD per gray for the $10 \mathrm{~Gy}$ film and approximately

3 Author to whom correspondence should be addressed. 
0.05 OD per gray for the 60 gray film. Klassen et al (1997) show a similar absorption spectrum for an MD-55-2 film but produce dramatically different results. For 6 Gy absorbed dose the response is approximately $0.1 \mathrm{OD}$ units per gray for $6 \mathrm{~Gy}$. This is approximately a factor of 2 higher at this specific wavelength. Not only is the change in optical density greater, the optical density of the non-irradiated film is much larger. As large variations in the sensitivity of the Gafchromic film are quoted in the literature, the authors wished to provide a simple method for increasing the sensitivity response of the Gafchromic film so that lower doses could be measured if required. A simple and straightforward way to increase the dose response for a detector using Gafchromic film is to use a layered detector. Thus using the principle described by the Beer-Lambert law (Pitt 1976) that the light absorbed by a medium varies exponentially with the path length of the light in the medium, we can effectively increase the change in optical density with absorbed dose by layering films together. The note aims to quantify the increases in the Gafchromic film absorption spectra for single-layer and multiple-layer detectors and to briefly analyse changes in spatial resolution caused by layered detectors.

\section{Materials and methods}

Measurements were performed on a Varian $2100 \mathrm{C}$ accelerator at photon energies of $6 \mathrm{MV}$ in a RMI $30 \times 30 \mathrm{~cm}^{2}$ solid water (Constanitinou et al 1982) slab phantom at $100 \mathrm{~cm}$ source-tosurface distance. The film used was Gafchromic MD-55-2 with batch number 970116 . Higher sensitivity film dosimeters were constructed using up to five layers of MD-55-2 films placed in a stack and stuck together with tape. The experimental results shown are the average of five measurements with errors quoted as one standard deviation of the mean. The film pieces were $1 \mathrm{~cm} \times 5 \mathrm{~cm}$ in size. The tape only covered the outer $1 \mathrm{~mm}$ edges of each dosimeter. Care was taken to make sure that the stack was bound tightly together, no air gaps were present between film layers and to reduce the effects of reflected/scattered light within the film stack and to minimize movement of the separate film pieces. This was confirmed by measurements of stack thicknesses compared to five individual layer thicknesses. Precautions in the handling of the radiochromic film outlined in TG-55 were used. The films during experiments storage and film analysis were kept at temperatures of $22 \pm 2{ }^{\circ} \mathrm{C}$ thus reducing the effects of timeand temperature-dependent evolution and readout (Meigooni et al 1996) of the absorption spectra of the film. The film was only removed from a light tight envelope during irradiation and readout to reduce the effects of ambient light (Butson et al 1998). Measurements were performed to analyse both the absorption sensitivity relationship with layer thickness and the spatial resolution of the film at various thicknesses. The film stacks were placed in the penumbral region of an x-ray beam for comparison of the spatial resolution for each layered detector. The film was analysed using two methods. The absorption spectra and sensitivity to dose of the radiochromic film were measured using a Shimadzu UV-160 UV-visible recording spectrophotometer. Its wavelength range is $200-1100 \mathrm{~nm}$ and is able to scan with a speed of up to $2400 \mathrm{~nm}$ per minute. The spectral bandwidth is $3 \mathrm{~nm}$, the wavelength readout $\pm 0.1 \mathrm{~nm}$ and has a wavelength accuracy of $\pm 0.5 \mathrm{~nm}$. It uses a double beam system and can measure optical density (OD) within 0.005 OD units. The spatial resolution of the film was measured using a modified Scanditronix densitometer, which uses a readout wavelength of $660 \pm 10 \mathrm{~nm}$ (FWHM) (Carolan et al 1997). The manufacturer quotes its spatial resolution as $0.1 \mathrm{~mm}$. Negligible polarization effects (Klassen et al 1997) were observed using this densitometer.

\section{Results and discussion}

Figure 1(a) shows the initial absorption spectrum for the non-irradiated Gafchromic film in a single layer and for a five-layer stack. Due to the absorption characteristics we can see that a 


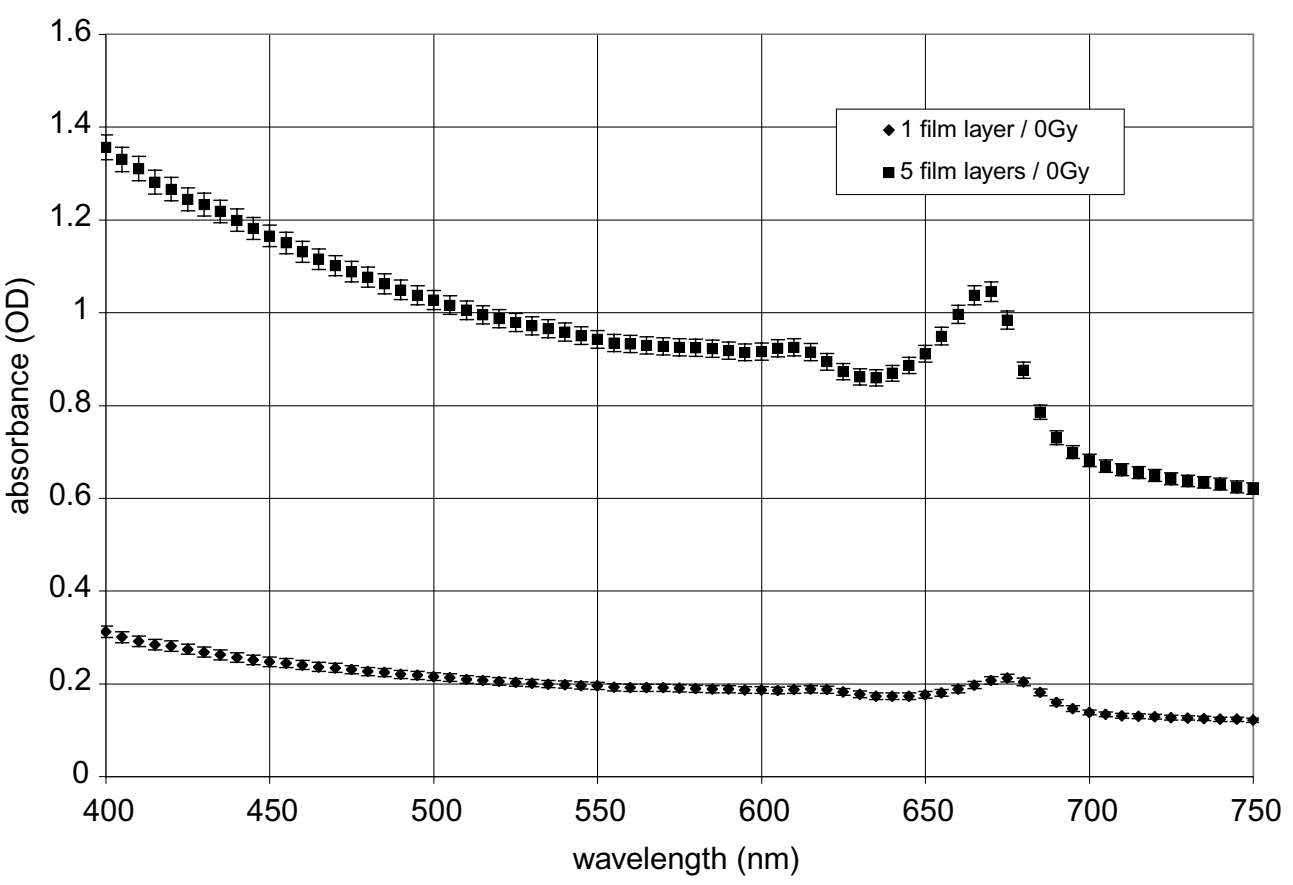

(a)

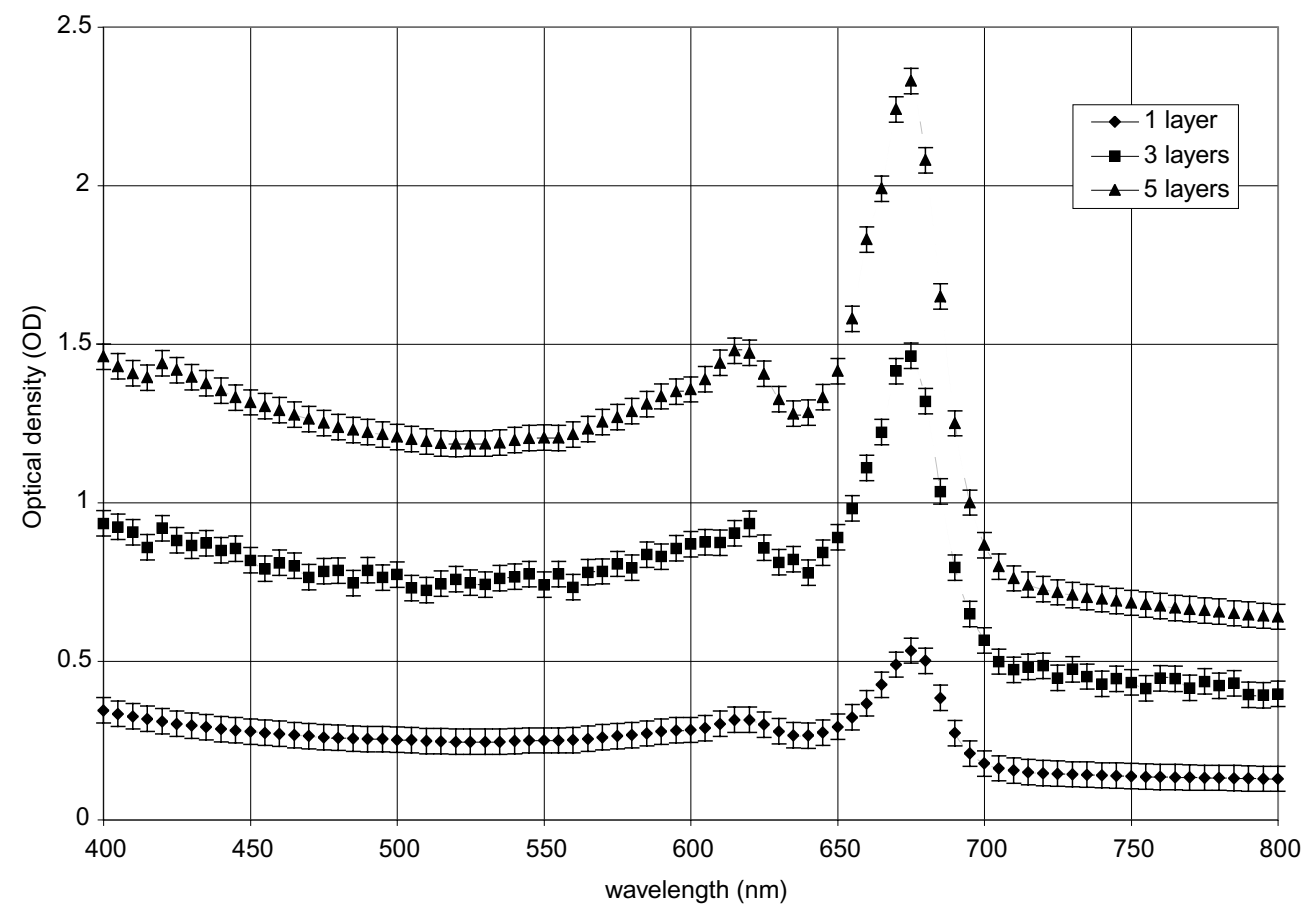

(b)

Figure 1. (a) Absorption spectra for an un-irradiated one-layer and five-layer MD-55 Gafchromic film detector. (b) Absorption spectrum for one, three and five layers of MD-55-2 Gafchromic film irradiated to 6 Gy applied dose. 


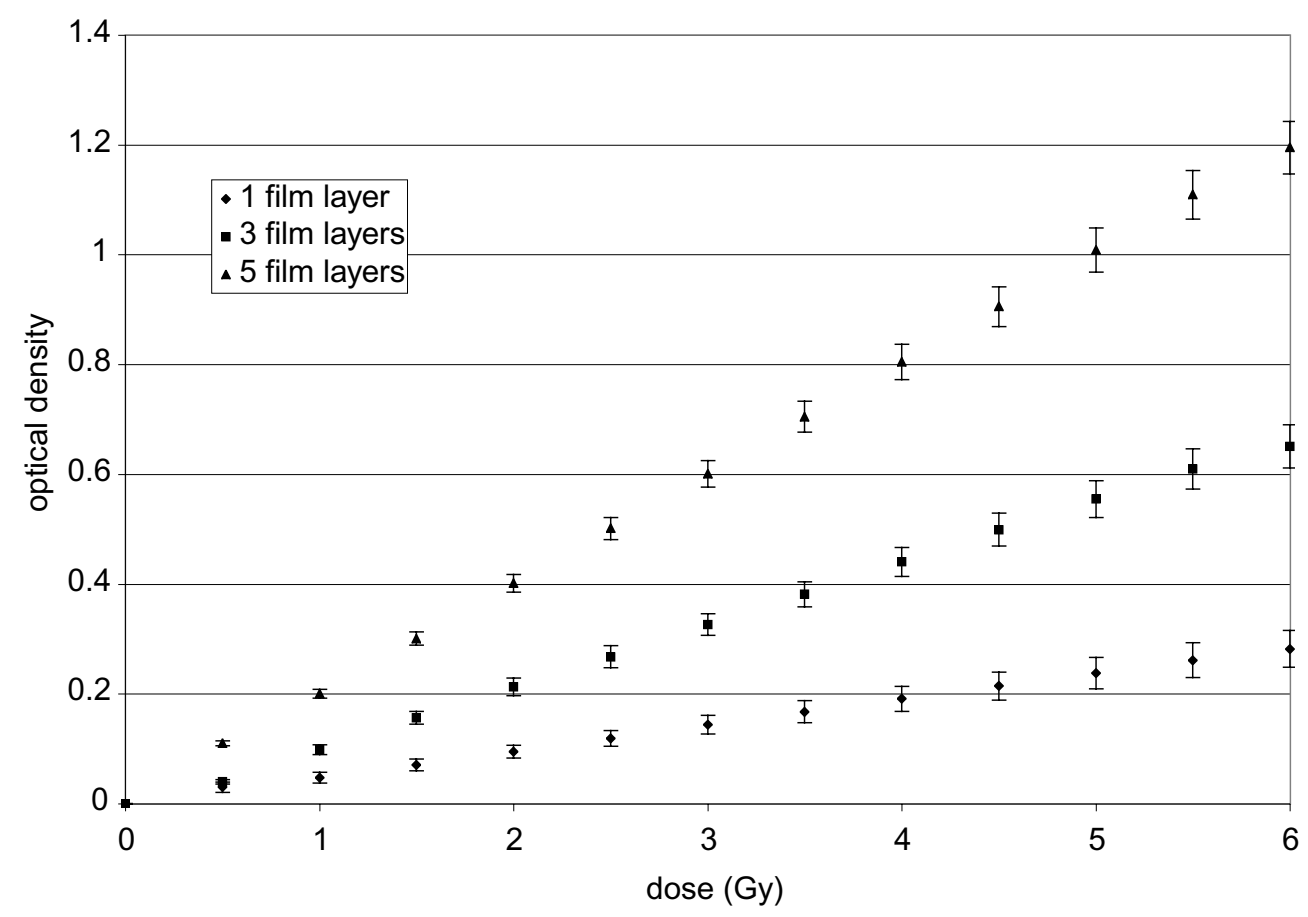

Figure 2. Increases in sensitivity measured at $670 \mathrm{~nm}$ for multi-layered film detectors.

substantial increase in absorbance has occurred at all wavelengths due to the additive nature of absorbance per unit thickness of material. Figure 1(b) shows an absorption spectrum for a single-layer, three-layer and five-layer Gafchromic film detector with a 6 Gy applied dose from a $6 \mathrm{MV}$ x-ray beam produced by a Varian 2100C linear accelerator. The single layer results match closely with McLaughlin et al's (1991) results for all wavelengths between 450 and $750 \mathrm{~nm}$. The three- and five-layer detector produced larger absorbances per unit-absorbed dose. Thus we can effectively increase the change in optical density per unit-absorbed dose by increasing the number of films stacked. To highlight the differences in relative optical density increase, figure 2 shows the relative net increase in optical density at a $670 \mathrm{~nm}$ wavelength readout for one, three and five film layers for doses up to $6 \mathrm{~Gy}$. From these results an increase in sensitivity of approximately $430 \%$ is seen for the five-layer dosimeter compared to the single layer film. Due to variations in the sensitivity response of film sheets and interbatch variability for the Gafchromic film, these results are only indicative of the increases in sensitivity. Each layered detector made would need a pre-exposure calibration to calculate the sensitivity response for that individual detector. This is the case for single layer films as well; thus a double exposure technique can be used for determining the dose response.

One area of concern is the possible reduction in the spatial resolution involved by using a layered dosimeter. Figure 3 shows results for a comparison of measurement in the penumbral region of a $6 \mathrm{MV}$ x-ray, $10 \mathrm{~cm} \times 10 \mathrm{~cm}$ field size at $1.5 \mathrm{~cm}$ depth. One-, three- and five-layer dosimeter results are shown. The measured 80: $20 \%$ penumbral width for the one-, threeand five-layer detectors is $3.1 \pm 0.3,3.3 \pm 0.3$ and $3.5 \pm 0.3 \mathrm{~mm}$, respectively. The errors quoted are one standard deviation for the five detectors measured. This represents a slight decrease in spatial resolution, which however, would not cause serious concern for typical 


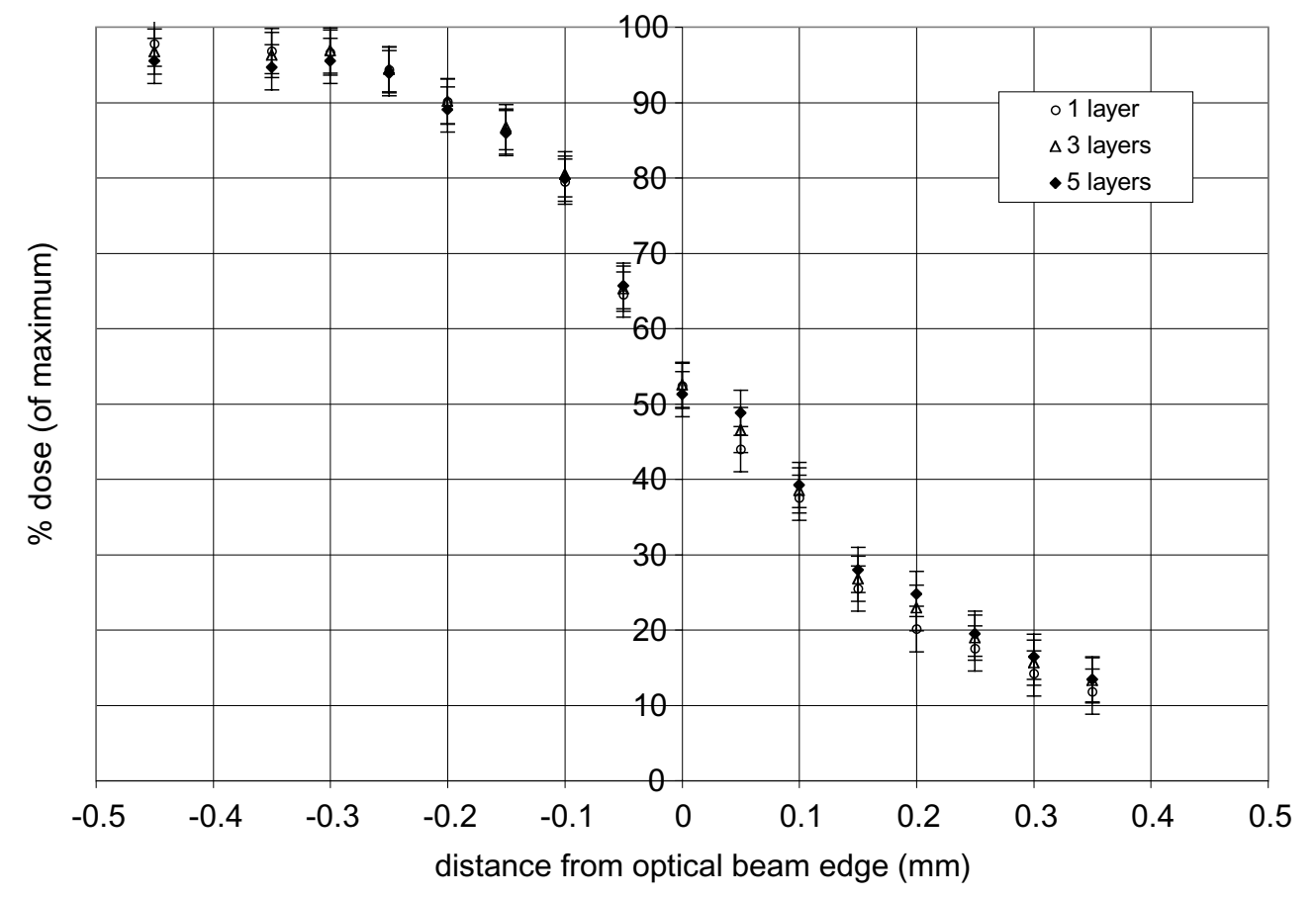

Figure 3. Penumbral measurements using one, three and five layers of radiochromic film. Results were measured with applied doses of 10,5 and $2 \mathrm{~Gy}$, respectively. The penumbral widths measured are 3.1, 3.3 and $3.5 \mathrm{~mm}$, respectively. The optical beam edge is defined as the position where the measured OD was closest to the $50 \%$ of maximum OD.

radiotherapy applications. The main reason for five-layer dosimeters being the maximum size tested in this work was due to the effective thickness of the detector becoming significant, $(0.84 \mathrm{~mm})$ producing a bulky detector which loses some ability to be shaped around a contour if necessary. If the film does not need to be shaped into any form, more layers of film could be used to further increase sensitivity.

\section{Conclusion}

Layered Gafchromic film detectors can be used to increase the sensitivity of a radiochromic film detector while not significantly diminishing spatial resolution. Tests were performed up to an arbitrary five layers; however, more layers could possibly be used to further increase sensitivity to the dose of a layered Gafchromic film detector if required.

\section{Acknowledgments}

This work has been fully supported by a grant from the Research Grants Council of HKSAR, China (Project no CityU 1137/00P). 


\section{References}

Butson M, Yu P and Metcalfe P 1998 Effects of readout light sources and ambient light on radiochromic film Phys. Med. Biol. 43 2407-12

Carolan M, Butson M, Herrmann K, Mathur J N and Metcalfe P 1997 Conversion of an infrared densitometer for radiochromic film analysis Australas. Phys. Eng. Sci. Med. $20185-9$

Constanitinou C, Attix F and Paliwal B 1982 A solid water phantom material for radiotherapy X-ray and gamma ray beam ray calculations Med. Phys. 9 436-41

Klassen N, Zwan L and Cygler J 1997 Gafchromic MD-55: investigated as a precision dosimeter Med. Phys. 24 1924-34

McLaughlin W, Yun-Dong C, Soares C, Miller A, Dyk G and Lewis D 1991 Sensitometry of the response of a new radiochromic film dosimeter to gamma radiation and electron beams Nucl. Instrum. Methods Phys. Res. A 302 165-76

McLaughlin W, Puhl J, Al Sheikhly M, Christou C, Miller A, Kovacs A, Wojnarovits L and Lewis D 1995 Novel radiochromic films for clinical dosimetry Proceedings of the 11th International Conference on Solid State Dosimetry II (Budapest)

1996 Novel radiochromic films for clinical dosimetry Radiat. Prot. Dosim. 66 263-8

Meigooni A, Sanders M, Ibbott G and Szeglin S 1996 Dosimetric characteristics of an improved radiochromic film Med. Phys. 23 1883-8

Niroomand-Rad A, Blackwell C, Coursey B, Gall K, Galvin J, McLaughlin W, Meigooni A, Nath R, Rodgers J and Soares C 1998 Radiochromic film dosimetry: recommendation of AAPM radiation therapy task group 55. Med. Phys. 25 2093-115

Pitt V 1976 Penguin Dictionary of Physics (Baltimore, MD: Penguin) 